\title{
Four mutations of the spastin gene in Japanese families with spastic paraplegia
}

\author{
Rehana Basri · Ichiro Yabe · Hiroyuki Soma · \\ Asako Takei · Hiroyuki Nishimura Y Yuka Machino · \\ Yasumasa Kokubo · Masafumi Kosugi · \\ Ryuichirou Okada · Motohiro Yukitake · \\ Hisao Tachibana $\cdot$ Yasuo Kuroda $\cdot$ Shigeki Kuzuhara \\ Hidenao Sasaki
}

Received: 28 February 2006/ Accepted: 7 April 2006/Published online: 21 June 2006

(C) The Japan Society of Human Genetics and Springer-Verlag 2006

\begin{abstract}
Hereditary spastic paraplegia (HSP) is a group of genetically heterogeneous neurodegenerative disorders characterized by slowly progressive spasticity and weakness of the lower limbs. HSP is caused by failure of development or selective degeneration of the corticospinal tracts, which contain the longest axons in humans. The most common form of HSP is caused by mutations of the spastin gene $(S P A S T)$, located on chromosome 2p21-p22, which encodes spastin, one of the ATPases associated with diverse cellular activities (AAA). In this study, we
\end{abstract}

Rehana Basri, Ichiro Yabe and Hiroyuki Soma have contributed equally to this work.

R. Basri · I. Yabe $\cdot$ H. Soma $\cdot$ H. Sasaki $(\square)$

Department of Neurology, Graduate School of Medicine,

Hokkaido University, Kita-ku, Sapporo 060-8368, Japan

e-mail: hsasaki@med.hokudai.ac.jp

A. Takei

Hokuyukai Neurology Hospital, Sapporo, Japan

H. Tachibana

Department of General Internal Medicine,

Hyogo College of Medicine, Nishinomiya, Japan

H. Nishimura

Department of Internal Medicine, Division of Neurology and Stroke Care Unit, Hyogo College of Medicine,

Nishinomiya, Japan

Y. Machino $\cdot$ Y. Kokubo $\cdot$ S. Kuzuhara

Department of Neurology, Graduate School of Medicine, Mie University, Tsu, Japan

M. Kosugi $\cdot$ R. Okada $\cdot$ M. Yukitake $\cdot$ Y. Kuroda Department of Neurology, Graduate School of Medicine, Saga University, Saga, Japan detected four causative mutations of SPAST among 14 unrelated patients with spastic paraplegia. Two missense mutations (1447A $\rightarrow \mathrm{G}, 1207 \mathrm{C} \rightarrow \mathrm{G}$ ) and two deletion mutations (1465delT, 1475-1476delAA) were located in the AAA cassette region. Three of these four mutations were novel. Previous reports and our results suggest that the frequency of SPAST mutations is higher among Japanese patients with autosomal dominant HSP, although SPAST mutations are also observed in patients with sporadic spastic paraplegia.

Keywords Spastic paraplegia $\cdot$ Spastin $\cdot S P A S T$. Hereditary spastic paraplegia

\section{Introduction}

Hereditary spastic paraplegia (HSP) is a group of clinically and genetically heterogeneous neurodegenerative disorders characterized by weakness, spasticity, and loss of vibration sensation in the lower limbs (Harding 1983). HSPs were conventionally classified on the basis of the pattern of inheritance, and by the presence (complicated HSP) or absence (pure HSP) of additional neurological features (e.g., mental retardation, epilepsy, optic atrophy, ataxia, amyotrophy, extrapyramidal features, sensory neuropathy, and retinopathy). HSP can be inherited in an autosomal dominant (AD-HSP), autosomal recessive (AR-HSP), or $\mathrm{x}$-linked recessive (X-HSP) fashion. Among these modes of inheritance, AD-HSP is the most common. To date, at least 29 genetic loci causing HSP have been identified, including 13 for AD-HSP, 13 for AR-HSP, and 3 for X-HSP (HUGO, http://www.gene.ucl.ac.uk/ 
nomenclature). Most families with AD-HSP (40\% or more) have mutations on chromosome 2p21-p22.

Hazan et al. (1999) reported that the spastin gene (SPAST) occupies approximately $90 \mathrm{~kb}$ of genomic DNA and contains 17 putative exons that encode spastin, a new member of the ATPases associated with the diverse cellular activities (AAA) protein family. The open reading frame encodes 616 amino acids, and there is marked similarity between the carboxy terminus (residues 342-599) of spastin and those of other AAA proteins. The members of this family are characterized by the possession of walker motifs $\mathrm{A}$ and $\mathrm{B}$, a AAA minimal consensus sequence, a leucine zipper motif, and a helix-loop domain (Sauter et al. 2002). The proteins act as molecular chaperones and play essential roles in many cellular activities, including cellcycle regulation, gene expression, and vesicle-mediated protein transport and protein degradation (Patel and Latterich 1998). More than 150 mutations of SPAST have been identified (Burger et al. 2000; Fonknechten et al. 2000; Tang et al. 2004; Depienne et al. 2006), including point mutations (missense, nonsense, and splice site), insertions, and deletions. It is noteworthy that SPAST mutations are more frequently associated with AD-HSP in Japanese patients (Namekawa et al. 2001; Namekawa et al. 2002; Yabe et al. 2002; Fukunaga et al. 2005; Iwanaga et al. 2005).

Here we present clinical and genetic data on new Japanese families with AD-HSP. In a previous report, Yabe et al. (2002) showed that 5 out of 12 probands with AD-HSP (41.6\%) had SPAST mutations. This time, we screened 14 unrelated Japanese patients, including 8 with AD-HSP and 6 with sporadic cases of unknown origin, to assess the frequency of the SPG4 gene. As a result, we identified four SPAST mutations, including three in patients with AD-HSP and one in a sporadic case. Three of these four mutations were novel.

\section{Materials and methods}

The clinical and genetic features of the 14 unrelated patients with spastic paraplegia $\{$ mean $\pm S D$ age: $51.67 \pm 4.51$ years, range $44-56$ years $\}$ were evaluated by neurologists after informed consent was given. Hokkaido University Ethics Committee approved this study. The affected individuals were selected using the Harding (1983) criteria for definition of clinical status, which include lower-limb spasticity in the absence of any evidence of structural lesions or demyelination. $\mathrm{AD}$ inheritance was present in eight cases and the remaining six cases had no family history. The procedure used for DNA sequence analysis has been described elsewhere (Yabe et al. 2002). In addition, a large deletion mutation of SPAST that was previously found in a Japanese family (Iwanaga et al. 2005) was analyzed by long-acting PCR (in a total volume of $20 \mu \mathrm{l}$ ) using an LA PCR Kit ver2.1 (Takara, Japan; catalogue RR013A). With this kit, it was possible to amplify large alleles up to about $48 \mathrm{Kbp}$. The PCR reaction mixture was initially denatured at $96^{\circ} \mathrm{C}$ for $2 \mathrm{~min}$, followed by 30 cycles of denaturation at $94^{\circ} \mathrm{C}$ for $40 \mathrm{~s}$, annealing at $51^{\circ} \mathrm{C}$ for $40 \mathrm{~s}$, and extension at $72^{\circ} \mathrm{C}$ for $3 \mathrm{~min}$. Final elongation was done at $72^{\circ} \mathrm{C}$ for $10 \mathrm{~min}$, after which LA-PCR products were separated by $1 \%$ agarose gel electrophoresis (the size of the normal allele was $3 \mathrm{Kbp}$ ).

\section{Results}

Four different mutations of SPAST were identified in 4 of the 14 unrelated SPG patients (Table 1). There were two missense mutations, including (1447A $\rightarrow \mathrm{G})$ in exon 11 and $(1207 \mathrm{C} \rightarrow \mathrm{G})$ in exon 7 , as well as two deletion mutations in exon 11 (1465delT and 1475de1AA). Three of these four mutations were novel. We detected these mutations in DNA of the affected individuals, but not in 50 normal controls (data not shown). No large deletion mutations were found in any of these patients. The clinical features of the four patients are summarized in Table 2. The initial symptom of all four patients was unsteadiness of the gait. Their cranial nerves and the muscle tone and power of the upper limbs were normal. Spinal MRI showed no abnormalities or atrophy of the spinal cord.

\section{Case 1}

A 56-year-old woman had developed gait unsteadiness starting at 8 years of age. At the age of 36 years, she noted difficulty with running. At the age of 50 years, she required aids for walking. Neurological examination revealed generalized hyperreflexia, bilateral sustained ankle clonus, diminished vibration sensation in the lower limbs, and a spastic gait. She was well oriented and had normal cognitive function without evidence of optic atrophy, nystagmus, or dysarthria. All of these neurological findings suggested that she had pure HSP. Her sibling also had a spastic gait and was mentally retarded. Her father died of colon cancer at the age of 82 years. He did not have gait disturbance. Her mother is still alive at the age of 84 years and does not have spastic paraparesis. 
Table 1 SPAST mutations in our patients with spastic paraplegia

\begin{tabular}{lllll}
\hline Patient no. & Exon location & Nucleotide change & Mutation type & Amino acid change \\
\hline 1 & Exon 11 & $1447 \mathrm{~A} \rightarrow \mathrm{G}$ & Missense & D441G \\
2 & Exon 11 & $1465 \mathrm{delT}$ & Frameshift & - \\
3 & Exon 7 & $1207 \mathrm{C} \rightarrow \mathrm{G}$ & Missense & P361R \\
4 & Exon 11 & $1475-1476$ delAA & Frameshift & - \\
\hline
\end{tabular}

Table 2 Clinical characteristics of our SPG patients

\begin{tabular}{|c|c|c|c|c|c|c|c|c|c|c|}
\hline \multirow{2}{*}{$\begin{array}{l}\text { Patient } \\
\text { no. }\end{array}$} & \multirow{2}{*}{$\begin{array}{l}\text { Family } \\
\text { history }\end{array}$} & \multirow[t]{2}{*}{ Age } & \multirow[t]{2}{*}{ Sex } & \multirow{2}{*}{$\begin{array}{l}\text { Age at } \\
\text { onset }\end{array}$} & \multirow[t]{2}{*}{ Disability } & \multicolumn{5}{|c|}{ Clinical features } \\
\hline & & & & & & $\begin{array}{l}\text { UL } \\
\text { hyperreflexia }\end{array}$ & $\begin{array}{l}\text { LL } \\
\text { hyperreflexia }\end{array}$ & $\begin{array}{l}\text { Ankle } \\
\text { clonus }\end{array}$ & $\begin{array}{l}\text { Vibration } \\
\text { sensation }\end{array}$ & Spasticity \\
\hline 1 & + & 56 & $\mathrm{~F}$ & 8 & 4 & + & + & + & Mild & + \\
\hline 2 & + & 52 & M & 49 & 2 & + & + & - & Normal & + \\
\hline 3 & - & 52 & M & 48 & 3 & + & + & + & Moderate & + \\
\hline 4 & + & 47 & $\mathrm{~F}$ & 39 & 3 & + & + & + & Mild & + \\
\hline
\end{tabular}

Disability was scored as 1 normal or very slight stiffness; 2 moderate gait disturbance; 3 unable to run, but able to walk; 4 able to walk with assistance; 5 wheelchair-bound. $U L$ Upper limb, $L L$ lower limb (Fonknechten et al. 2000)

Case 2

A 52-year-old man first noted gait unsteadiness and difficulty in bending his legs at the age of 49 years, which had progressed over 3 years. Neurological examination revealed generalized hyperreflexia (particularly in the lower limbs) and a spastic gait. Other neurological findings (such as vibration sensation in the lower limbs) were normal. His mother had developed gait disturbance at the age of 55 years, and required assistance at 75 years, while his brother (48 years old) had similar neurological symptoms. The clinical phenotype of this family was pure HSP without complicating features such as optic atrophy or sensory impairment.

\section{Case 3}

A 52-year-old man presented to the Neurology Department with a history of unsteady gait and frequent falls for 3 years. Neurological examination revealed generalized hyperreflexia, lower-limb spasticity, bilateral ankle clonus, and diminished vibration sensation in the lower limbs, but no extensor plantar reflex. Other family members had no neurological diseases. His father had died at the age of 86 years, and his mother had died at 68 years. Neither of them had gait disturbance or any other neurological problems.

\section{Case 4}

A 47-year-old woman had first noted weakness of the lower limbs and difficulty bending her legs from the age of 39 years. Currently, she has difficulty running and climbing stairs, but can walk unaided. Neurological examination revealed generalized hyperreflexia, bilateral spasticity, and diminished vibration sensation in the lower limbs. There was increased ankle clonus, but no extensor plantar reflex. Her father, an older sister, and one elder brother also had similar neurological problems, with the age at onset being 46, 48, and 49 years, respectively. Her cognitive function was normal and there was no evidence of optic atrophy.

\section{Discussion}

In this study, three new mutations of SPAST were detected. All of these new mutations affected the highly conserved coding region of the AAA cassette. The two novel deletion mutations (1465delT and 147576delAA) led to frameshift and a premature termination codon, resulting in the production of a truncated protein. We also detected two missense mutations $(1447 \mathrm{~A} \rightarrow \mathrm{G}$ and $1207 \mathrm{~A} \rightarrow \mathrm{G})$ in the highly conserved AAA cassette coding region, and one of these $(1207 \mathrm{~A} \rightarrow \mathrm{G})$ was novel. The $1447 \mathrm{~A} \rightarrow \mathrm{G}$ mutation results in the replacement of aspartic acid with glycine at position 441 , while $1207 \mathrm{C} \rightarrow \mathrm{G}$ leads to replacement of proline with arginine at position 361. Mutations of the AAA cassette coding region may lead to loss of protein activity (Patel and Latterich 1998). Most of the SPAST missense mutations within the AAA domain of $S P A S T$ alter the ability of the protein to regulate interactions with its target and thus lead to constitutive binding to microtubules (Errico et al. 2002). Associa- 
tion of spastin with the microtubule cytoskeleton is mediated by the $\mathrm{N}$-terminal region of this protein and is regulated through the ATPase activity of the AAA domain (Reid et al. 2003). It was recently demonstrated that spastin is a microtubule-severing enzyme and that several disease-associated mutations impair its enzymatic activity (Evans et al. 2005). Impairment of the fine regulation of the microtubule cytoskeleton in long axons by mutations of SPAST may underlie the pathogenesis of HSP. Unfortunately, we could not confirm cosegregation with the disease and obtain clinical information on other members of our families since we did not have their cooperation. However, these mutations were considered to be critical because they were located in the AAA cassette and could not be found in healthy controls.

In a previous study, no common mutations were identified. Therefore, allelic heterogeneity of SPAST was assumed to contribute to variability in the clinical phenotype of SPG patients, although some AD-HSP families had SPAST mutations linked to $2 \mathrm{p}$ (Fonknechten et al. 2000; Tang et al. 2004). Recently, Depienne et al. (2006) reported that genetic, epigenetic, and environmental factors could also influence disease expression in carriers of sporadic SPAST mutations. In our study, we identified one patient (case 3) who had a sporadic SPAST mutation and no family history. In such circumstances, we provide genetic counseling to the affected individuals.

All of the patients in this series had pure spastic paraplegia without other neurological findings, reticular changes, cataract, or skin disease. Previous reports have described that SPAST mutations most often cause pure HSP, although there are exceptions (Tallaksen et al. 2001, Proukakis et al. 2003). However, it was recently demonstrated that patients with SPG4linked AD-HSP and an average age of 67 years showed a mean 9-point decrease in their Cambridge cognitive examination (CAMCOG) score over a 3-year period (McMonagle et al. 2004). In contrast, there was no evidence of definite cognitive impairment or dementia in our HSP patients with SPAST mutations, even in the sporadic case.

We previously reported (Yabe et al. 2002) five Japanese families with SPAST mutations who represented $41.6 \%(5 / 12)$ of the AD-HSP cases in that series. In addition, we found new SPAST mutations accounting for $37.5 \%$ (3/8) of our current AD-HSP cases. Only one patient (case 3) had the SPAST mutation associated with sporadic spastic paraplegia. The present findings suggest that structural SPAST mutations account for approximately $30-50 \%$ of AD-HSP in Japanese families. Iwanaga et al. (2005) reported one patient who had a large deletion involving the $5^{\prime}$-UTR of $S P A S T$, and we also investigated their large deletion mutation in our cases. However, we could not rule out other large deletion mutations other than this one because direct sequencing was not suitable to analyze them (Sauter et al. 2002). Although the frequency of large deletion mutations of $S P A S T$ is unknown, it is possible that the frequency of $S P A S T$ mutations in Japanese patients with AD-HSP would be increased somewhat by detecting such mutations. We also found a SPAST mutation in a sporadic elderly patient with moderate spasticity. Some of the mutations occurring in sporadic cases might have a lower penetrance than those identified in families (Depienne et al. 2006). In addition, the variety of mutations in our cases indicated that each pedigree had a different founder.

In summary, we reported four SPAST mutations that caused AD-HSP in Japanese patients. Testing patients with spastic paraplegia for SPAST mutations, even in the absence of a positive family history, may provide more information about the functions of spastin protein.

Acknowledgments We are very grateful to the present families for their willingness to participate in this study. This work was partly supported by a Grant-in-Aid for the Research Committee for Ataxic Diseases of the Research on Measures for Intractable Diseases from the Ministry of Health, Welfare and Labour, Japan; by a Grant-in-Aid for the Research Committee for CNS Degenerative Diseases of the Research on Measures for Intractable Diseases from the Ministry of Health, Welfare and Labour, Japan; by a Grant-in-Aid for Scientific Research from the Ministry of Education, Science, Sports, and Culture, Japan; by a grant from the Akiyama Foundation; and by a grant from the Noastic Foundation.

\section{References}

Burger J, Fonknechten N, Hoeltzenbein M, Neumann L, Bratanoff E, Hazan J, Reis A (2000) Hereditary spastic paraplegia caused by mutations in the SPG4 gene. Eur J Hum Genet 8:771-776

Depienne C, Tallaksen C, Lephay JY, Bricka B, Poea-Guyon S, Fontatine B, Labauge P, Brice A, Durr A (2006) Spastin mutations are frequent in sporadic spastic paraparesis and their spectrum is different from the one observed in familial cases. J Med Genet 43:259-265

Errico A, Ballabio A, Rugarli EI (2002) Spastin, the protein mutated in autosomal dominant hereditary spastic paraplegia, is involved in microtubule dynamics. Hum Mol Genet 11:153-163

Evans KJ, Gomes ER, Reisenweber SM, Gundersen GG, Lauring BP (2005) Linking axonal degeneration to microtubule remodeling by spastin-mediated microtubule severing. J Cell Biol 168:599-606

Fonknechten N, Mavel D, Byrne P, Davoine C, Cruaud CS, Boentsch D, Samson D, Coutinho P, Hutchinson M, McMonagle P, Burgunder JM, Tartaglione A, Heinzlef $\mathrm{O}$, Feki I, Deufel T, Parfrey N, Brice A, Fontaine B, 
Prud'homme JF, Weissenbach J, Durr A, Hazan J (2000) Spectrum of SPG4 mutations in autosomal dominant spastic paraplegia. Hum Mol Genet 9:637-644

Fukunaga S, Ikezoe K, Ohyagi Y, Taniwaki K, Kira J, Morita M (2005) A case of sporadic SPG4 with leu 534 pro (Japanese). Rinsho Shinkeigaku 45:618

Harding AE (1983) Classification of the hereditary ataxias and paraplegias. Lancet 21:1151-1155

Hazan J, Fonknechten N, Mavel D, Paternotte C, Samson D, Artiguenave F, Davoine CS, Cruaud C, Durr A, Wincker P, Brottier P, Cattolico L, Barbe V, Burgunder JM, Prud'homme JF, Brice A, Fontaine B, Heilig R, Weissenbach J (1999) Spastin, a new AAA protein, is altered in the most frequent form of autosomal dominant spastic paraplegia. Nat Genet 23:296-303

Iwanaga $\mathrm{H}$, Tsujino A, Shirabe S, Eguchi H, Fukushima N, Niikawa N, Yoshiura KI, Eguchi K (2005) Large deletion involving the $5^{\prime}$-UTR in the spastin gene caused mild phenotype of autosomal dominant hereditary spastic paraplegia. Am J Med Genet 133A:13-17

McMonagle P, Byrne P, Hutchinson M (2004) Further evidence of dementia in SPG4-linked autosomal dominant hereditary spastic paraplegia. Neurology 62:407-410

Namekawa M, Takiyama Y, Sakoe K, Nagaki H, Shimazaki H, Yoshimura M, Ikeguchi K , Nakano I, Nishizawa M (2002) A Japanese SPG4 family with a novel missense mutation of the SPG4 gene: intrafamilial variability in age at onset and clinical severity. Acta Neurol Scand 106:387-391
Patel S, Latterich M (1998) The AAA team: related ATPase with diverse functions. Trends Cell Biol 8:65-71

Proukakis C, Auer-Grumbach M, Wagner K, Wilkinson PA, Reid E, Patton MA, Warner TT, Crosby AH (2003) Screening of patients with hereditary spastic paraplegia reveals seven novel mutations in the SPG4 (Spastin) gene. Hum Mutat 21(2):170-174

Reid E (2003) Science in motion: common molecular pathological themes emerge in the hereditary spastic paraplegias. J Med Genet 40:81-86

Sauter S, Miterski B, Klimpe S, Bonsch D, Schols L, Visbeck A, Papke T, Hopf HC, Engel W, Deufel T, Epplen JT, Neesen J (2002) Mutation analysis of the spastin gene (SPG4) in patients in Germany with autosomal dominant hereditary spastic paraplegia. Hum Mutat 20:127-132

Tallaksen CM, Durr A, Brice A (2001) Recent advances in hereditary spastic paraplegia. Curr Opin Neurol 14:457-463

Yabe I, Sasaki H, Tashiro K, Matsuura T, Takegami T, Satoh T (2002) Spastin gene mutation in Japanese with hereditary spastic paraplegia. J Med Genet 39:e46 